\title{
Conductance Quantization in a Periodically Modulated Quantum Channel: backscattering and mode mixing
}

\author{
P. Singha Deo1 ${ }^{1 \text {, B. B. C. Gupta }}{ }^{2}$, A. M. Jayannavar ${ }^{2}$, and F. M. Peeters ${ }^{1, \text { 日 }}$ \\ 1 Dept. of Physics, University of Antwerp (UIA), Universiteitsplein 1, \\ B-2610 Antwerpen, Belgium. \\ 2 Institute of Physics, Bhubaneswar 751005, India
}

\begin{abstract}
It is known that the conductance of a quantum point contact is quantized in units of $2 e^{2} / h$ and this quantization is destroyed by a non-adiabatic scatterer in the point contact, due to backscattering. Recently, it was shown [Phys. Rev. Lett. 71, 137 (1993)] that taking many non-adiabatic scatterers periodically in a quantum channel, the quantization can be recovered. We study this conductance quantization of a periodic system in the presence of a strong defect. A periodic arrangement of double-stubs give remarkable quantization of conductance. A periodic arrangement of double-constrictions also gives a very good quantization only when the separation between the constrictions is small. We conclude that conductance quantization of a periodically modulated channel is robust.
\end{abstract}

PACS numbers :72.10.-d,73.20.Dx 


\section{INTRODUCTION}

In a novel experiment van Wees et al [1] and Wharam et al [2] found that the conductance of a nano-meter scale constriction between two 2D electron gas increases monotonically in steps of $2 e^{2} / h$ as more and more propagating sub-bands become available. Such a constriction is referred to as a quantum point contact (QPC). This quantization of conductance is due to the lateral quantization of the energy (due to the formation of sub-bands) and sheds light on the physical relevance of Landauer's formula [3]. Transport along the wire with such quantized states become reflection-less due to the absence of backscattering and then the conductance quantization is simply evident from the Landauer conductance formula. Energy can also be quantized by applying a large magnetic field. In such a situation the four probe Hall conductance is found to be quantized and the phenomenon is well known as the Integral Quantum Hall Effect (IQHE) [四]. Whereas in IQHE the quantization has been observed to an accuracy of one part in billion, the accuracy in the QPC experiments is one part in hundred. This departure was therefore taken very seriously. Since then the conductance of QPC has been studied in great details, experimentally as well as theoretically. Various aspects like the role of point defects in the point contact (PC) [5], the role of evanescent modes in the PC [0, 6], mode matching at the junction of the contact and the reservoir [7], adiabatic as well as non-adiabatic shape changes of the point contact [8,9], non-uniformity of potential in th PC [10], bound states in the presence of non-adiabatic shape changes [11 13], periodic modulations in the point contact [9, 14, 16], magnetic field in the PC [17], etc. have been studied in details. Non-adiabatic constrictions in the point contact roughly maintain the quantization [8], but if there is a single non-adiabatic cavity in the point contact then this quantization is completely washed away [9]. Recently Leng and Lent [18] have shown that the quantization of the conductance is recovered if a large number of non-adiabatic constrictions are taken in the point contact periodically, however, the conductance does not increase monotonically with the energy. It was analyzed by Leng and Lent [18] that back scattering can be suppressed in these periodically modulated chan- 
nels (except in the narrow regions between resonances which can not be avoided in a finite periodic system) because the periodicity results in Bloch state like good quantum states and thus resulting in a perfect conductance quantization. However, the periodic system has to be connected to leads on both sides, and due to matching of wave-function between the leads and the periodic system there will be some mixing of the transverse modes (modes arising due to the finite thickness in the direction perpendicular to the propagating direction). In the presence of some channel mixing it is possible that all the resonances in the transmission probabilities are not due to the symmetry of the system but some of them are Fano resonances, arising due to the mixing of transverse modes [5]. Hence, if there are defects or disorder in the system, which will result in the breakdown of the symmetry of the system, it is possible that this may not affect the Fano resonances much, although it will obviously affect the resonances arising due to the symmetry. Hence, it may be interesting to see the effect of a defect on the quantized conductance of a periodically modulated channel.

Earlier we have reported [19] the effect of a defect on the conductance of a periodically modulated channel when only one propagating sub-band is populated in the main wire and we showed that the defect has very drastic effects on the conductance which can be used for efficient band-tailoring. In this paper we study the effect of similar defects on the conductance when two or more sub-bands are occupied.

This paper is organized as follows. In section II we outline the numerical technique used. In section III we graphically discuss our results. In section IV our conclusions are summarized. And finally section $\mathrm{V}$ is devoted to acknowledgments.

\section{THEORETICAL TREATMENT}

Fig. 1 shows a single non-adiabatic geometric scatterer in a point contact. The point

contact is bounded between $-\frac{b}{2}<\mathrm{x}<\frac{b}{2}$ whereas the scatterer in the point contact is bounded between $-\frac{c}{2}<\mathrm{y}<\frac{c}{2}$. The width of the scatterer in the $x$ direction is $2 a$. When $c>b$ we refer to the system as a double-stub whereas for $c<b$ we refer to the system 
as a double-constriction. The scatterer is symmetric in the $y$ direction. So the sub-bands will have a definite parity and opposite parity states can never mix. By cascading many such units we can make a periodically modulated QPC or quantum channel. Reservoirs are attached to two ends of the sample. We do not consider any mode matching at the junctions of the reservoirs. As a result we do not have evanescent modes in the main wire but we do have sufficient number of evanescent modes inside the non-adiabatic scatterer. In a physical situation where the leads connecting the sample to the reservoirs become large, the effect of this mode matching becomes unimportant [16. And moreover, if we include evanescent modes in the main wire, the system of periodic arrangement of double stubs and the system of periodic arrangement of double-constrictions become similar to each other. We deliberately make them different to give us some physical insight.

We have evaluated the conductance or the transmission coefficient across the system numerically and the procedure followed is briefly outlined below. The details can be found in Ref. [20]. The system is unbounded in the propagating direction, i.e., the $x$ direction whereas in the $y$ direction the system is bounded due to the finite width. The hard wall boundary conditions are assumed along the boundaries of the channel and these conditions define the channel modulation. Inside the system we have taken the magnitude of the quantum mechanical potential $V=0$, i.e., we are in the classically unbound regime. We first consider the case of a single scatterer as shown in Fig. 1. The two dimensional Schrödinger equation for the system is (we set $\hbar=2 m=1$ )

$$
\nabla^{2} \psi(x, y)=E \psi(x, y)
$$

where $E$ is the total energy of an electron and $\psi$ is the wave function. In the leads far away from the scatterer the electron propagates in a perfect narrow wire where eigenstates can be decoupled into a longitudinal plane wave part $e^{i k_{m} x}$ and a transverse part $\psi_{m}(y)$. The wave function $\psi_{m}(y)$ corresponds to motion between infinite potential walls along the $y$ direction is

$$
\psi_{m}(y)=\sqrt{\frac{2}{b}} \sin \left[\frac{m \pi}{b}(y+b / 2)\right], \quad m=1,2,3 \ldots
$$


The scattering states are of the form

$$
\psi_{m}^{L}(x, y)=\frac{e^{i k_{m} x}}{\sqrt{ } k_{m}} \sin \left[\frac{m \pi}{b}(y+b / 2)\right]+\sum_{n=1}^{\infty} R_{n m} \frac{e^{-i k_{n} x}}{\sqrt{ } k_{n}} \sin \left[\frac{n \pi}{b}(y+b / 2)\right] \quad \text { for } x \leq-a,
$$

and

$$
\psi_{m}^{R}(x, y)=\sum_{n=1}^{\infty} T_{n m} \frac{e^{i k_{n} x}}{\sqrt{ } k_{n}} \sin \left[\frac{n \pi}{b}(y+b / 2)\right] \quad \text { for } x \geq a
$$

where the index $n$ runs over all possible transverse modes. The incident particle is in the $m$-th mode. The longitudinal wave vectors are given by

$$
k_{n}^{2}=E-n^{2}\left(\frac{\pi}{b}\right)^{2}
$$

$E$ being the total energy. The quantity $n^{2} \pi^{2} / b^{2}$ is the energy associated with the $n$-th transverse mode and $k_{n}^{2} / 2 m$ is the energy associated with the longitudinal motion of an electron in the $n$-th transverse mode.

The transmission $\left(T_{n m}\right)$ and reflection $\left(R_{n m}\right)$ amplitudes are to be determined by matching solutions across $|x|=a$ and can be expressed in terms of the matrix coefficients

$$
\begin{gathered}
R_{m m^{\prime}}=\left|\frac{S_{m m^{\prime}}^{e}+S_{m m^{\prime}}^{o}}{2}\right|^{2}, \\
T_{m m^{\prime}}=\left|\frac{-S_{m m^{\prime}}^{e}+S_{m m^{\prime}}^{o}}{2}\right|^{2},
\end{gathered}
$$

where

$$
S_{m, m^{\prime}}^{e}=e^{-i k_{m} a}\left[1+2 i\left(F^{e}-i\right)^{-1}\right]_{m, m^{\prime}} e^{-i k_{m^{\prime}} a}
$$

and

$$
S_{m, m^{\prime}}^{o}=e^{-i k_{m} a}\left[1+2 i\left(F^{o}-i\right)^{-1}\right]_{m, m^{\prime}} e^{-i k_{m^{\prime}} a}
$$

The matrices $F^{e}$ and $F^{o}$ are obtained by matching the wave-function outside the scatterer to those inside the scatterer and are given by (for details see Ref. [20])

$$
F_{m^{\prime} m}^{e}=-\frac{b}{c\left(k_{m^{\prime}} k_{m}\right)^{1 / 2}} \sum_{p=1}^{\infty} I_{m^{\prime} p} I_{m p} K_{p} \tan \left(K_{p} a\right)
$$


and

$$
F_{m^{\prime} m}^{o}=-\frac{b}{c\left(k_{m^{\prime}} k_{m}\right)^{1 / 2}} \sum_{p=1}^{\infty} I_{m^{\prime} p} I_{m p} K_{p} \cot \left(K_{p} a\right)
$$

where

$$
I_{m n}=\frac{2}{\pi}\left[\frac{\sin \left[\left(\frac{m}{b}-\frac{n}{c}\right) \frac{\pi b}{2}\right] \cos \left[\frac{(m-n) \pi}{2}\right]}{m-\frac{n b}{c}}-\frac{\sin \left[\left(\frac{m}{b}+\frac{n}{c}\right) \frac{\pi b}{2}\right] \cos \left[\frac{(m+n) \pi}{2}\right]}{m+\frac{n b}{c}}\right],
$$

and $K_{p}$ is the wave vector along the $x$ direction associated with the $p$-th channel in a doublestub. The sums in Eqs.(9) and (10) run over infinite values of $p$. This is because in the interior region of the scatterer, infinite number of states (one from each transverse mode) couple to the electronic state of incident energy $E$. Some of them are propagating modes and some of them are evanescent modes. The sums in Eqs.(9) and (10) are highly converging and one can truncate them appropriately depending on the accuracy required. Typically 10 evanescent modes are included in our calculations.

Having found the complex transmission and reflection amplitudes across one such scatterer we use the scattering matrix cascading technique to find the transmission across many such scatterers put together in series. The separation between the two scatterers is $l$ and all other lengths $b, a, c$ associated with the scatterer are the same as in Fig. 1. For details of the method of cascading we refer to Ref. [18]. Here we just outline the algorithm.

The scattering matrix $(\mathrm{S})$ for any symmetric scatterer is given by

$$
\left(\begin{array}{ll}
r & t^{\prime} \\
t & r^{\prime}
\end{array}\right)
$$

where the $r$ and $t$ are matrices:

$$
r=\left(\begin{array}{cccc}
R_{11} & R_{12} & \ldots & R_{1 n} \\
\vdots & \vdots & \ddots & \vdots \\
R_{n 1} & R_{n 2} & \ldots & R_{n n}
\end{array}\right)
$$

and

$$
t=\left(\begin{array}{cccc}
T_{11} & T_{12} & \ldots & T_{1 n} \\
\vdots & \vdots & \ddots & \vdots \\
T_{n 1} & T_{n 2} & \ldots & T_{n n}
\end{array}\right)
$$


For the case of symmetric scatterers considered in our work we have $t^{\prime}=t$ and $r^{\prime}=r$. There are $n$ incoming channels and $n$ outgoing channels. If two scatterers are characterized by the $\mathrm{S}$ matrices $S_{1}$ and $S_{2}$ then the resultant scattering matrix, on cascading the two systems, is the direct product of $S_{1}$ and $S_{2}$. The total S matrix of N scatterers is the direct product of $\mathrm{N}$ scattering matrices. The order in the direct product of the N S-matrices must be kept the same as that of the spatial order of the scatterers. This is because the $\mathrm{S}$ matrices do not commute. Thus from the total S matrix we can find the two terminal-conductance $\mathrm{G}$, which is given by the Landauer formula [3] i.e., $\mathrm{G}=\frac{2 e^{2}}{h} \sum_{i j}\left|T_{i j}\right|^{2}$.

\section{RESULTS AND DISCUSSIONS}

We first consider the system of a quantum channel (main wire) in which a series of double-constrictions are arranged periodically. The system under consideration consists of 87 double-constrictions. We have taken $a / b=0.5, c / b=0.805$. The distance between two consecutive constrictions is $l / b=0.5$ where $b$ is the width of the main wire along the $y$ direction. Here, $2 a$ and $c$ are the widths of a single constriction along the $x$ and $y$ direction,

respectively. The dimensionless conductance, $\mathrm{g}\left(=\frac{h}{2 e^{2}} G\right)$ for this system is plotted in Fig. 2 (dotted curve) as a function of dimensionless incident energy $E b_{1}^{2}$ (where $b_{1}=b / 2$ is the half width of the main wire). We vary the incident energy in the range such that two to seven propagating modes in the main wire are occupied. We find a conductance quantization which is not monotonically increasing but the quantization is not good. At higher energies, since there are a lot of propagating modes, there are a lot of nearly degenerate channels and small mode mixing can wash out the quantization. And at lower energies, in the case of such a large value of $2 a(2 a / l=1)$, there are not many Fano resonances in the system to produce a good quantization. The reason is that the exponentially decaying evanescent modes have a negligible probability density inside the constriction. A breakdown of the symmetry by the presence of a defect can completely destroy the quantization as demonstrated in Fig. 2 (solid curve). We replace the middle constriction of the original system by a different constriction. 
The width of the newly placed constriction (defect constriction) is such that $c / b=0.905$. Such a defect in a QPC is a non-adiabatic or a very strong scatterer. Other parameters are the same as for the other constrictions. Fig. 2 (solid curve) shows the dimensionless conductance $(g+3)$ as a function of the dimensionless incident energy for this system. The incident energy is varied to excite two to seven modes in the main wire. We see that the quantization of the conductance is lost except for some of the lowest steps of the few lowest sub-bands. Thus a single defect is enough to destroy the quantization of the conductance of the system.

We can increase the probability density of the modes in the scatterer, i.e., by taking a double-stub or a very small double-constriction. In case of the double-stub there will be more propagating modes in the scatterer and in case of the short double-constriction the evanescent modes in the constriction will have a large probability density. In the following we demonstrate that in these two cases we get very good quantization and also they are robust even in the presence of the defect.

First we consider a system comprising of a series of identical double-stubs arranged periodically in a point contact. We take 87 double-stubs. Other parameters for this system are given by $a / b=0.5$ and $c / b=1.055$. The distance between two consecutive doublestubs is $l / b=0.5$. Here, $2 a$ and $c$ are the widths of a single double-stub along the $x$ and $y$ directions, respectively. Fig. 3 (dotted curve) shows the dimensionless conductance for this system as a function of dimensionless incident energy. In this case we see that the quantization is very good. So, we see that a system consisting of a series of double-stubs in a point contact gives better conductance quantization than that of a series of constrictions. This is in contrast to the fact that a single double-stub in a QPC destroys quantization more effectively than a single constriction [8]. We now put a defect in the system. The defect is introduced by the replacement of the middle double-stub in the system by another one whose width in the $y$ direction, taken to be $c / b=1.205$, is different from the other double-stubs. We excite up to seven modes in the main wire by a proper choice of the range of incident energy. The dimensionless conductance $(g+3)$ for this system (the system with 
a defect) is plotted as a function of dimensionless incident energy. This is shown in Fig. 3 (solid curve) where we notice that the quantization of the conductance still partly survive and is not destroyed completely. Quantization is still retained up to the 4th or 5th step. Backscattering in some regimes is negligibly small as found by comparing the solid curve with the dotted curve in Fig. 3.

Next, in Fig. 4 (dotted curve) we consider a system that consists of 87 small doubleconstrictions regularly placed in a main wire. We have taken $a / b=0.125$ (so it is a small constriction), $c / b=0.805$, and the distance between two consecutive constrictions is $l / b=$ 0.5. Note that the only difference in this case, i.e., the value of $a$, is very small compared to the system considered in Fig. 2. Again we restrict ourselves to maximum seven mode propagation. Here we see that the quantization of the conductance is much better than that in Fig. 2. We have examined the quality of quantization of conductance for systems with various values of $a / b$ and noticed that the quality of the quantization of conductance degrades as the the value of $a / b$ deviates from 0.125 such that $a / b$ and $l / b$ are incommensurate. Now we introduce a defect constriction in the system as just discussed in the above in order to simulate mode mixing and backscattering. We replace the middle constriction by a defect constriction. The value of $c / b$ for the defect constriction is 0.905 while the other parameters are the same as for the other constrictions. The dimensionless conductance $(g+3)$ of the system is plotted as a function of dimensionless incident energy in Fig. 4 (solid curve). Two to seven propagating modes are considered. We notice that the quantization of conductance for the lower bands are fairly retained even in the presence of this defect.

\section{CONCLUSIONS}

The quantization of conductance in periodic channels is more robust than that in unmodulated point contacts. The experiment of Kouwenhoven et al [14] measures the conductance of a periodic channel but one has to take a longer chain to see the quantization. But because of the robustness of the effect it will be worthwhile to do such an experiment in a 
long channel. A system of periodic stubs is more robust than that of a system of periodic constrictions. But if the spacing between the constrictions is made very small, then the quantization of periodic constrictions is equally robust. The Fano resonances, that are always present in a multichannel scattering problem, have a strong influence on the quantization of the conductance.

\section{ACKNOWLEDGMENTS}

One of us (PSD) thanks Prof. N. Kumar for useful discussions. This work is supported by the Flemish Science Foundation(FWO-Vl) grant No: G.0277.95, and the Belgian InterUniversity Attraction Poles (IUAP-VI). One of us (PSD) is supported by a scholarship from the University of Antwerp and FMP is a Research Director with FWO-Vl. 


\section{REFERENCES}

* email address: deo@uia.ua.ac.be

o email address: peeters@uia.ua.ac.be

[1] B. J. van Wees, H. van Houten, C. W. J. Beenakker, J. G. Williamson, L. P. Kouwenhoven, D. van der Marel, and C. T. Foxon, Phys. Rev. Lett. 60, 848 (1988).

[2] D. A. Wharam, T. J. Thornton, R. Newbury, M. Pepper, H. Ahmed, J. E. Frost, D. G. Hasko, D. C. Peacock, D. A. Ritchie, and G. A. C. Jones, J. Phys. C 21, L209 (1988).

[3] R. Landauer, Z. Phys. B 68, 217 (1978).

[4] T. Chakraborty and P. Piettilienen, The Fractional Quantum Hall Effects, Berlin; Springer Verlag (1988).

[5] P. F. Bagwell, Phys. Rev. B, 41, 10354 (1990).

[6] K. F. Bergrenn and Z. Ji, Phys. Rev. B, 43, 4760 (1991); ibid. 45, 6650 (1992).

[7] G. Kirczenow, Solid State Commun. 68, 715 (1988).

[8] A. Szafer and A. D. Stone, Phys. Rev. Lett. 62, 300 (1989).

[9] Hua Wu, D. W. L. Sprung, J. Martorell and S. Klarsfeld, Phys. Rev. B 44, 6351 (1991).

[10] E. Tekman and S. Ciraci, Phys. Rev. B. 43, 7145 (1991) and references therein.

[11] F. M. Peeters, Science and Engineering of one and zero Dimensional Semiconductors, Vol. 214 of NATO Advanced Study Institute Series B Physics, eds. S. P. Beaumont and C. M. Sotomayor-Torres (Plenum Press, New York, 1990), p.107.

[12] A. Nixon, J. H. Davies, and H. U. Baranger, Phys. Rev. B. 43, 12638 (1991).

[13] P. N. Butcher and J. A. McInnes, J. Phys. Cond. Mat. 7, 745 (1995).

[14] L. P. Kouwenhoven, F. W. J. Hekking, B. J. van Wess, C. J. P. M. Harmans, C. E. 
Timmering, and C. T. Foxon, Phys. Rev. Lett. 65, 361 (1990).

[15] J. A. Brum, in Proc. of 20th International conference on The Physics of Semiconductors, Vol.3, ed. E. M. Anastassakis and J. D. Joannopoulos, World Scientific, Singapore, p.2363.

[16] R. Akis, P. Vasilopoulos and P. Debray, Phys. Rev. B 52, 2805 (1995).

[17] J. U. Nöckel, Phys. Rev. B 45, 14225 (1992).

[18] M. Leng and C. S. Lent, Phys. Rev. Lett. 71137 (1994); M. Leng and C. S. Lent, Phys. Rev. B 50, 10832(1994).

[19] P. Singha Deo and A. M. Jayannavar, Physica B 228, 3531996.

[20] B. F. Bayman and C. J. Mehoke, Am. J. Phys. 51, 875 (1983).

\section{Figure captions}

Fig.1. Schematic diagram of a Quantum point contact with a non-adiabatic scatterer in it. The axes directions and the various length parameters are shown in the figure.

Fig.2. (dotted curve) Plot of dimensionless conductance (g) versus dimensionless Fermi energy $\left(E b_{1}^{2}\right)$ for a system of 87 double-constrictions $(a / b=0.5, c / b=0.805, l / b=0.5)$. (solid curve) Plot of dimensionless conductance (g) versus dimensionless Fermi energy $\left(E b_{1}^{2}\right)$ for a system that has a defect at the center of the system considered in the dotted curve. For the central double-constriction $c / b=0.905$.

Fig.3. (dotted curve) Plot of dimensionless conductance (g) versus dimensionless Fermi energy $\left(E b_{1}^{2}\right)$ for a system of 87 double-stubs $(a / b=0.5, c / b=1.055, l / b=0.5)$. (solid curve) Plot of dimensionless conductance $(\mathrm{g})$ versus dimensionless Fermi energy $\left(E b_{1}^{2}\right)$ for a system that has a defect at the center of the system considered in the dotted curve. For the central double-constriction $c / b=1.205$.

Fig.4. (dotted curve) Plot of dimensionless conductance (g) versus dimensionless Fermi energy $\left(E b_{1}^{2}\right)$ for a system of 87 double-constrictions $(a / b=0.125, c / b=0.805, l / b=0.5)$. 
(solid curve) Plot of dimensionless conductance (g) versus dimensionless Fermi energy $\left(E b_{1}^{2}\right)$ for a system that has a defect at the center of the system considered in the dotted curve. For the central double-constriction $c / b=0.905$. 


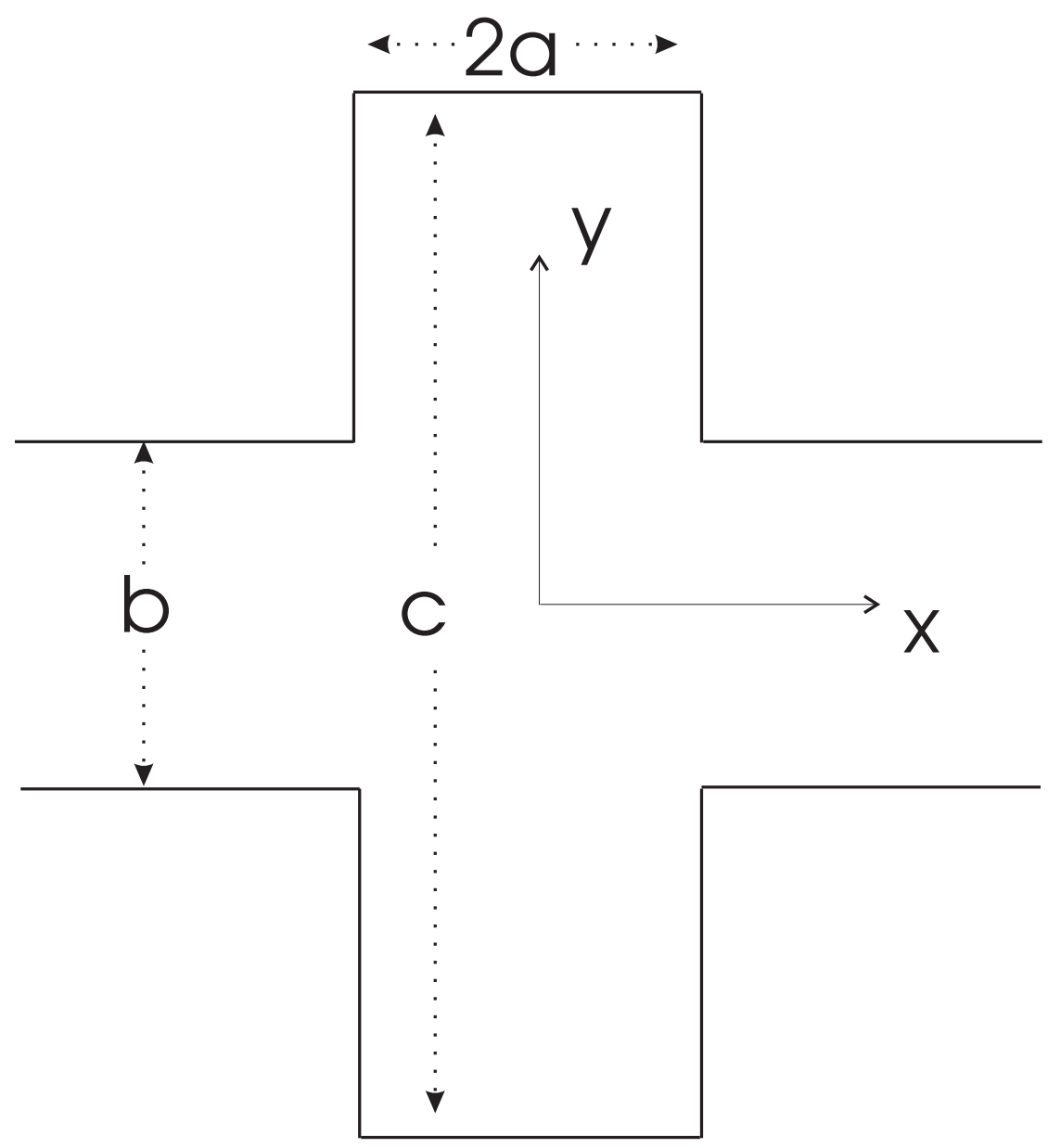




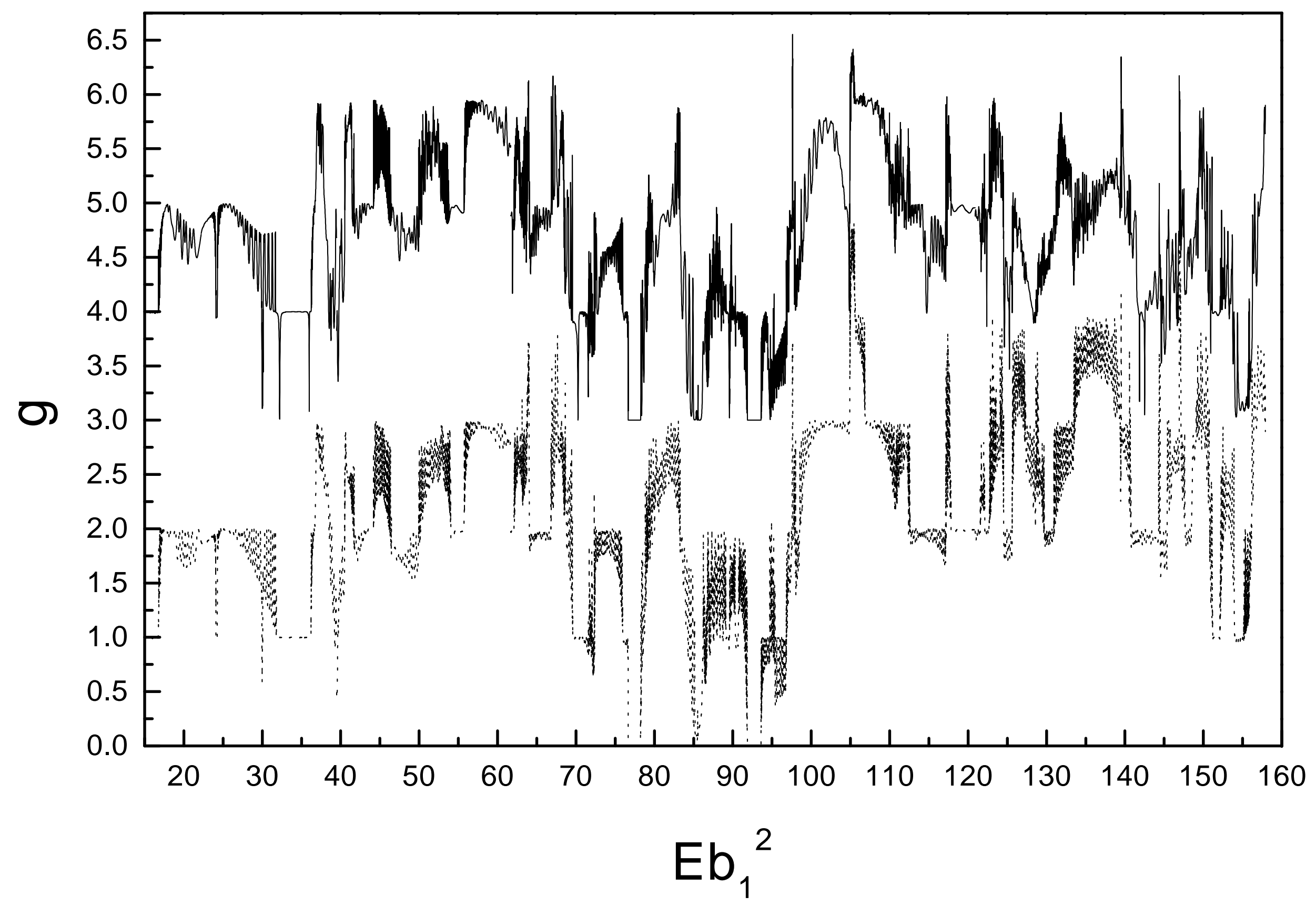




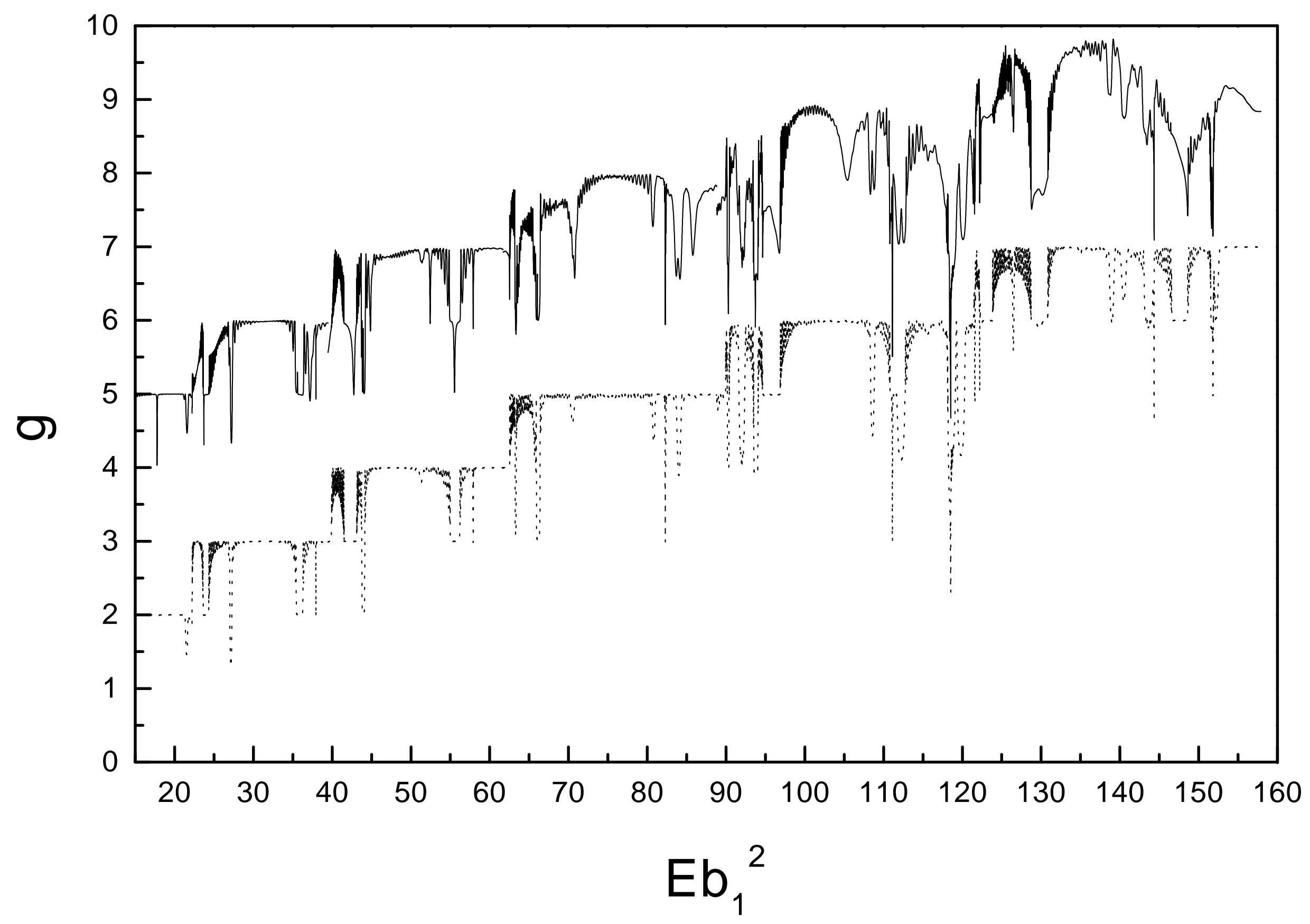




$$
\text { reprympy }
$$

- 4 of 